\title{
Variability due to Contact Configurations
}

\author{
HORST MAUDER (Bamberg)
}

The question of whether W UMa stars are contact binaries or not is still a problem. It is promising to see that the theoretical investigators are on the way to explain that and how contact binaries can really exist, even at age zero and with different masses. Let us assume - as is generally done - that the surfaces of the two components of a W UMa star can be set identical to the equipotential surfaces of the Roche model of two mass points. If these surfaces coincide with the limiting Roche lobe or even exceed it for a little amount, than we are justified to call the figure a contact system. However, it may well be that one star fills its critical lobe and the other one is a little bit below it, or even both components may be below their limiting lobes by a small amount. The uncertainty of the elements that can be derived from W UMa light curves for the time being does not allow for a definite decision. We have to look, therefore, for the possible differences between the light-curves of very close, not contact binaries and the variability of contact pairs.

If we look at the geometrical properties of the limiting Roche lobe we see, that there is a remarkable difference of this configuration, in the quadratures, relative to a pair of two detached, ellipsoidal stars. However, things change appreciably if we go on from the quadratures towards the eclipses. At the onset of eclipse the shapes of the two components, as seen from an observer, are not very different from ellipsoids, and they become so even more, as the eclipse proceeds. It is especially for this reason why the normal Russell-Merrill solution workes rather good even for W UMa stars and often gives results which are in good agreement with the spectroscopic data. The most remarkable difference in the shape of a contact configuration and the normal Russell-Merrill model appears in the vicinity of the inner Lagrangian point. Especially during the eclipses there should be very little difference. Can we hope to decide the question of whether $\mathrm{W}$ UMa stars are contact systems or not from any evidence of the light curves? It was shown by MAUDER (1969) and by SCHÖFFEL (1970), that the light changes of rotating contact configurations, not taking into account the effect of the eclipses, show a remarkable difference against the normal behaviour of a pair of ellipsoidal stars. This is in the sense, that the coefficient $A_{2}$ in the Fourier series expansion of the continuous light changes is larger outside the eclipses than inside. Applying this to the actual, observed light curves of W UMa stars yield a remarkable result: If the normal solution procedure is applied on W UMa stars one gets (at best) a set of solution parameters. From this it is possible to calculate a theoretical light curve and to compare this with the observations. Now it turns out that observations and theoretical curve fit rather well - except for the phases directly after onset of the eclipse and immediately before its end. At this interval the observed points are systematically fainter than the theoretical ones. This disagreement does not occur if the light changes due to the contact model are applied. Unfortunately, this is not a crucial test for the contact hypothesis since the light changes are due to both components. If the light changes outside the eclipses are dominated by the more massive, brighter component, than it is sufficient that the primary fills its limiting lobe, but the secondary may still be a little bit below its critical surface. To complicate things still further, MERRILL (1970) was able to show, that the same behaviour of $A_{2}$, though less pronounced, may occur for distorted, not ellipsoidal stars, which are a little bit below the limiting surface. This was applied by BOOKMYER (1969) to the system V 566 Oph. It is evident, however, that the best hance for a solution of the problem will come from a neat discussion and calculation of the light changes outside of the eclipse and from the phases shortly after first and before fourth contact. To do this it is urgently needed to know something about the distribution of brightness over the surface of the stars, since the normal laws for limb-and gravity-darkening are probably insufficient to describe the situation on the facing hemispheres of the two components. It can be hoped that the results as presented by BIERMANN and THOMAS or those given by LUCY (1968) will allow in the near future 
a better theoretical explanation of the structure of those systems. For the time being, with respect to W UMa stars, we should to the statement .Variability due to Contact Configurations" still add a questionmark.

$$
\text { Ref ere n ces: }
$$

BOOKMYER, B. B., 1969, Astronomical J. 74, 1197.

LUCY, L. B., 1968, Astrophys. J. 151, 1123 and 153, 877.

MAUDER, H., 1969, IAU Coll. Budapest, 419.

MERRILL, J. E., 1970, in "Vistas in Astronomy“, Vol. 12, 43.

SCHOFFEL, E., 1970, Veröff. Bamberg, 8, 88.

\title{
Discussion to the paper of MAUDER
}

THOMAS: If W UMa systems are not in contact, how do you think the energy can be transferred from one component to the other?

MAUDER: I have no physical model for this, but as you know, most light curves of W UMa stars suffer some variability. Therefore, due to the thermal energy content of matter it might well be, that occasional mass exchange might transport the excess energy radiated away by the secondary.

HAZLEHURST: Did you imply that the surface brightness may be higher in the region between the stars?

MAUDER: No, I only mentioned, that in this region the normal expressions for limb- and gravity-darkening might well fail to be applicable since those laws are valid only in the case of hydrodynamic equilibrium.

\section{Search for Contracting Close Binaries}

\author{
R. H. KOCH (Philadelphia)
}

The selection of close binaries which compose the usual tabulations of solutions has informed us in detail on main sequence stellar atmospheric and interior parameters, and on the theories of the tidal and radiative interactions, and has permitted the creation of the theory of binary evolution. As we have seen, the eruptive doubles are beginning to yield to modern instrumentation. Adding more pairs to those already "understood" will bring continuity into our appreciations of these processes and undoubtedly change them sowewhat. The origins of close pairs, however, remain obscure and one can only say that the theories which describe fission of a proto-star after its core de-couples from the envelope seem promising. The latest presentation of this idea by BODENHEIMFR and OSTRIKER (1970) can be viewed as the successor to the indicative theories of ROXBURGH (1966) and CAMERON (1969). In this way of looking at binary origins it is simply a question of when fission occurred in the proto-stellar interval. Observationally, candidate stars are anything but numerous and it is for this reason that we have begun the program described here.

More than 50 plates have been taken of NGC 2264 primarily with the No. $191-\mathrm{cm}$ reflector at the Kitt Peak National Observatory. Kodak IIa D emulsion was used and the beam was filtered by a cemented sandwich of $1 \mathrm{~mm}$. BG 18 and of $2 \mathrm{~mm}$. GG 495 A. This gives a response very like the familiar $V$ sensitivity with an effective wavelength perhaps $100 \AA$ shorter than that for $V$. The five consecutive nights from Kitt Peak used the photoelectrically-guided photographic tailpiece but were of inferior photometric quality. Exposure times were of the order of 20 minutes. Lengthened exposures which attempted to compensate 